\title{
Research on programming and constructing of Microgrids which aim at absorbing clean energies
}

\author{
Cheng Liu ${ }^{1, a^{*}}$, Chun Chen ${ }^{1, b}$, Hui Wang ${ }^{1, \mathrm{c}}$ and Xiaoyan Zhang ${ }^{1, d}$ \\ ${ }^{1}$ NO.19 Chengxin Road, Jiangning District, Nanjing City, Jiangsu Province, China \\ aliucheng2@sgepri.sgcc.com.cn, ${ }^{b}$ chenchun@sgepri.sgcc.com.cn, \\ cwang-hui2@sgepri.sgcc.com.cn, ${ }^{d}$ zhangxiaoyan@sgepri.sgcc.com.cn
}

\begin{abstract}
Keywords: clean energy, absorb, Microgrid
Abstract. With the fast development of clean energies, absorbing cleaning energies becomes a critical problem to solve while the economy develops and society progresses. This paper presents a new type of Microgrid by analyzing methods of enhancing clean-energy-absorbing ability. The new type of Microgrid could efficiently enhance clean-energy-absorbing ability, raise clean energy permeability, which leads to development of clean energy construction and national economy.
\end{abstract}

\section{Overview}

In recent years in China clean energies develop fast. By the end of year 2016, the installed capacity of clean energies in State Grid is 440 million $\mathrm{kW}$, in which water power is 216 million $\mathrm{kW}$, wind power is 132 million $\mathrm{kW}$ and solar power is 72.06 million $\mathrm{kW}$. International Energy Agency(IEA) forecasts that by the year 2020, two third of global power supply increment will be new energies, and by the year 2025 new energy will be the second biggest power source in the world.

While the installed capacity increases, abandoned wind and solar power increases. Annual utilization hours of device decrease, and in some areas because the grid cannot absorb the power, abandoning of wind, solar and water power becomes more and more, which becomes the biggest problem that affects healthy development of clean energies.

The Microgrid, as the most efficient active distribution network (ADN) component which could raise the reliability and stability of power supply forms, is also an important technique to realize smart grid [1]. To satisfy the needs of absorbing clean energies, promote using of clean energies, reduce smog, the Microgrid needs innovation in both form and structure.

Based on traditional Microgrid, this paper considers the needs of absorbing clean energies, discusses and improves the composition, structure and operating principle of Microgrid, analyzes the way to enhance clean-energy-absorbing ability, presents a new type of Microgrid, which could efficiently enhance the absorbing ability and permeability of clean energies. This kind of Microgrid has high reliability and work efficiency, which indicates the developing direction of clean energies and Microgrid in the future.

\section{Key technique of the new Microgrid}

High Voltage Class. One important cause for low clean-energy-absorbing capability is low power delivering capability, which makes State Grid promote ultra-high voltage power transmission. Building high-voltage-and-wide-range Microgrid is a feasible way to solve the problem of difficulty in absorbing clean energies. Traditional Microgrid usually works on $400 \mathrm{~V}$ or $10 \mathrm{kV}$ voltage class, while the new Microgrid works on $35 \mathrm{kV}$ voltage class. Higher voltage class relates to bigger clean energy covering area, which could reduce losses during transmitting power, make coordinated controls to clean energies in a big area, and realize the complementary use of energies to enhance the absorbing capability of clean energies in the Microgrid.

Energy Converting Technique. Considering the diversity and complexity of energy-consuming ways in civil, commercial and industrial utilization, using energy converting technique between different energies can increase the absorbing rate of clean energies, and avoid the accumulation of clean energies. 
In 2013 State Grid proposed an electric power replacement scheme: electricity for coal, electricity for oil, and electricity comes from distant places. This scheme conduces to improve air quality. On the other hand, it uses electric power increment to promote clean energy absorbing. Meanwhile, electric power replacement increases electric power consuming, eases difficulties in absorbing clean energies and power system in peak load shaving in some areas in China[2].

Promoting electricity instead of coal and using of electric vehicles are ways to realize electricity for coal and electricity for oil. Ground Source Heat Pump (GSHP) technique is also one kind of electric power replacement technique.

Making respond to kinds of user need in the Microgrid, using energy converting techniques which are represented by electric power replacement, can efficiently enhance the absorbing capability of clean energies.

Peaking modulation capability of Microgrids. Currently lack of peaking modulation capability of clean energies is a critical cause for abandoning of wind, solar and water power.

It is discovered that by the year 2020, even if there is new-constructed direct current transmission pipeline, the abandoned rate of wind and solar power is over $20 \%$ in Xinjiang and Gansu province in China, and the electricity limitation rates is over $10 \%$ in Ningxia province. If there was no new direct current transmission pipeline, the abandoned rate of wind and solar power in Gansu would be near $50 \%$, which would be about $35 \%$ in Xinjiang. Simulations on peak modulation capability and transmission capacity show that compared between the year 2015 and 2020, the influence brought by lack of peak modulation capability is far bigger than that brought by limitation of transmission capacity.

Wind and solar power are generated with big intermittency and volatility. With the proportion of wind and solar power in Microgrid getting increased, the electric grid faces bigger and bigger challenge. Electric power sources with powerful peak modulation are needed to cooperate with wind and solar power generation in order to insure that power system work steadily and economically.

Pumped storage power station can store potential energy of water from spare electric power when electric load is in valley, it converts potential energy of water to electric power when load is in peak, which realizes effect electric power storage, and redistribute electric power in different time. As a result, dynamic balance between generation, supplying and consuming of electric power system is adjusted. Meanwhile, pumped storage power station has several characteristics like quick start up, flexibly changed operation condition, peak load shifting, frequency modulation-phase modulation, emergency use, etc, because of which it is called adjuster, voltage stabilizer or accumulator. Fuel power generation has similar functions like pumped storage power station.

To absorb clean energies adequately, when clean energies like wind and solar are connected to the Microgrid, we also construct fuel power generations or pumped storage power stations to adjust output power of Microgrids and enhance the peak modulation capability.

Storage System. As a necessary part in new energy generation, storage system can realize demand side management, eliminate difference between peak and valley, smooth the load, increase operation rate of device, decrease spinning reserve capacity, improve power quality, stabilize system, lower power supply cost, thus brings big benefit[3].

Meanwhile storage system in Microgrids can smooth output power curve, coordinate Microgrid output power, assist microgrids to do peak load shifting to the electric grid, and enhance the peak modulation capability of the Microgrid.

To enhance the absorbing capability of clean energies, battery pack with high energy density and big capacity should be chosen in the Microgrid.

\section{Framework of the new Microgrid}

Main framework. The new Microgrid works on $35 \mathrm{kV}$ voltage class, which is not a conventional working voltage class for loads, as a result we need to step up the voltage class. The new Microgrid takes layered and distributed framework as common Microgrids take. But because of the high voltage class and vast territory, we add a coordinating and dispatching layer above the master station layer to 
realize coordination in the Microgrid and dispatching of power and energy between the Microgrid and the electric grid. The framework of the Microgrid is shown in Fig.1.

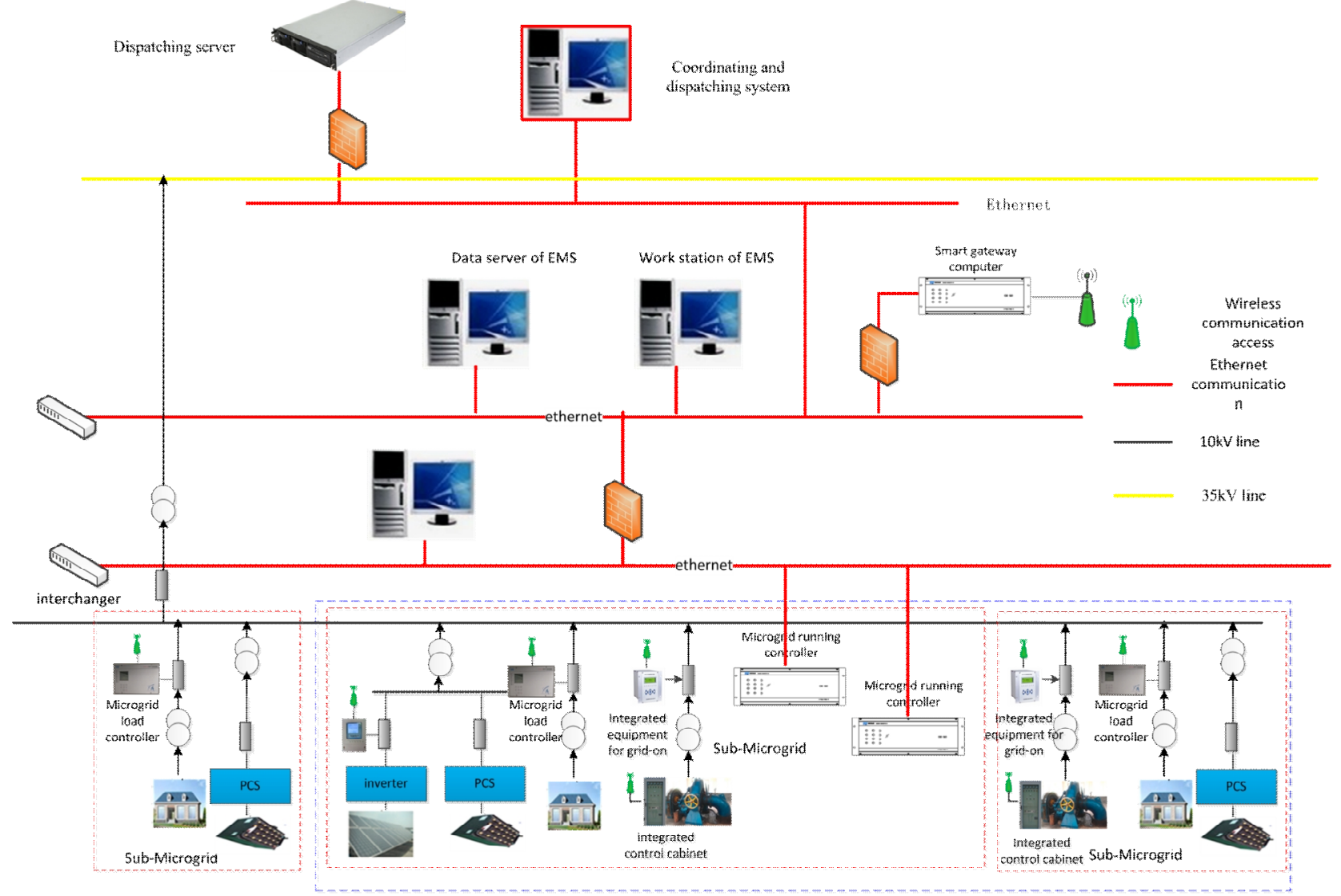

Fig. 1 Framework of the new Microgrid

Since the new Microgrid works on a high voltage class and covers a vast territory, the subordinate takes sub-Microgrids as active units. In Fig. 1 there are 3 sub-Microgrids. Sub-Microgrids work on $400 \mathrm{~V}$ or $10 \mathrm{kV}$ voltage class, working voltage classes of which are stepped up to $35 \mathrm{kV}$ to connect to each other.

In communication management, the coordinating and dispatching system communicates with energy management systems (EMS) of sub-Microgrids via optical fiber to make a total coordination control and communicates with the dispatching system of the electric grid to coordinate the power and energy control between the Microgrid and electric grid.

Software framework of the new Microgrid. The main part in the software system of the new Microgrid is dispatching and energy management system. Dispatching and energy management system uses advanced distribution automation platform framework, which satisfies the technical request for dispatching and energy managing of Microgrids. By collecting real time running data of sub-Microgrids, giving controlling instructions to sub-Microgrids according to established running and controlling strategy, the dispatching and energy management system ensures that the Microgrid and electric grid run safely and steadily. It could also make data analyses for Microgrid running.

The function framework of dispatching and energy management system is shown in Fig.2.

The function framework is divided into 4 layers: local layer, running and controlling layer, sub-Microgrid layer and dispatching and energy management system layer. Compared with traditional Microgrid framework, the new Microgrid has a dispatching and energy management system layer, which takes charge of energy coordinating and dispatching in the Microgrid, coordinated controlling between sub-Microgrids, energy control between Microgrid and the electric grid, energy and power output control of the Microgrid.

Hardware components of the Microgrid. Hardware system of the new Microgrid is also divided into 4 layers: local layer, running and controlling layer, sub-Microgrid layer and dispatching and energy management system layer. Local layer usually contains distributed generation, storage system, load controller, etc. 
Running and controlling layer is core of the Microgrid system, which takes charge of communicating of running data, executing of running and controlling strategy in the Microgrid. Running and controlling layer mainly contains Microgrid running controller, communication management controller, monitoring and governing equipment for power quality, etc.

Sub-Microgrid layer takes charge of energy controlling and optimized dispatching of

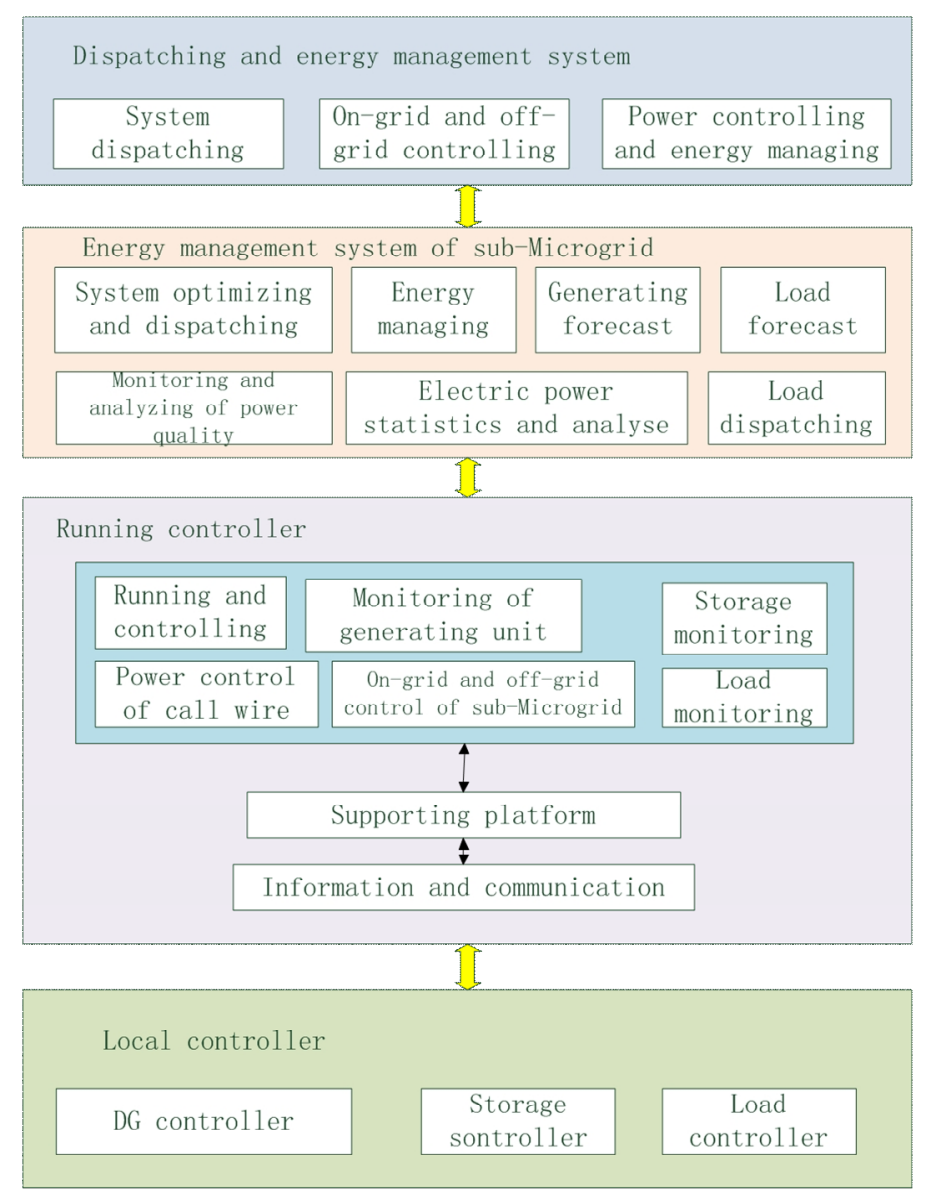

Fig.2 Function framework of dispatching and energy management system sub-Microgrids, which contains server,workstation, telecontrol machine, etc.

Dispatching and energy management system layer takes charge of coordinating energy flow management and communication between sub-Microgrids, it also controls the output power and on-grid and off-grid of the Microgrid. The hardware of this layer is usually made up of servers and workstations.

\section{Research on construction of the new Microgrid}

Site selection. Since the new Microgrid works on a high voltage class, and electric power sources and loads usually work on $400 \mathrm{~V}$ class, the Microgrid has high requirements for the electric grid, which calls for abundant structure and strong net rack. Furthermore, considering the requirements for energy converting technique and peak modulation capability of the Microgrid, the requirements for diversity of loads and distributed generation in the Microgrid are high.

Capacity determining. In Microgrid management method by China's National Energy Administration, the micro of Microgrid is defined as this: low voltage class, usually below $35 \mathrm{kV}$; small system scale not over 20MW, usually smaller than MW class.

Considering the limitation to wire diameter of power transmission line and transmission capacity, and the distance between sub-Microgrids, each sub-Microgrid should not have too big capacity, which is appropriate in MW class. If the sub-Microgrid has a big capacity, the power transmission line may be overburdened, the circuit may need remoulding. 
Capacities of power sources, storage systems and loads should be determined by actual generating ability and needs.

Running and Controlling strategy. According to the user needs and request of local policy and situation, variety of running and controlling strategies can be made. By different aims like economic running, absorbing clean energies, steady running, the new Microgrid can run following different strategies to get different effects.

\section{Summary and Outlook}

Recent years because of the needs of environmental governance and promotion of government policies, generation of clean energies has been greatly developed. But because of the lack of well-organized planning, construction and instruction, absorbing of clean energies has been quite difficult. In this paper through research on principle and method to enhance the ability of absorbing clean energies, considering structure of mainstream Microgrids, we propose a new type of Microgrid which satisfies the needs for absorbing clean energies. This new Microgrid can effectively enhance the ability of absorbing clean energies in Microgrid and promote the use of clean energies.

With the development of techniques and changing of policies and situation, utilizing method of clean energies will get developing. As a result, the new type of Microgrid proposed in this paper would also be improved, able to satisfy users' different needs.

\section{Acknowledgements}

This work was financially supported by the 2017 State Grid Scientific Project Research and Application on enhancing the absorbing ability and security technology of electric grid in photovoltaic poverty relief zone.

\section{References}

[1] Meijun Liu, Electric Power Science and Technology, Vol.27(2015), p.200(in Chinese)

[2] Zhen Zhang, Chinese Economy and Trade magazine, Vol.18(2016), p.51-52(in Chinese)

[3] Lili Wei, Qing Huang, Chunyan Liu, Qin Luo, et al. Electric Power Source Technology, Vol.3(2015), p.637-639(in Chinese) 